\title{
The Comparison of Three Assessment Scales in a Neonatal Unit in Poland
}

\author{
Aleksandra Tabaczyńska ${ }^{1}$, Roksana Malak ${ }^{1}$, Brittany Fechner $^{1}$, \\ Ewa Mojs ${ }^{2}$, Włodzimierz Samborski ${ }^{1}$, Ewa Baum ${ }^{3}$ \\ 1 Department and Clinic of Rheumatology, Rehabilitation and Internal Diseases, Poznan \\ University of Medical Science, Poland \\ 2 Department of Clinical Psychology, Poznan University of Medical Sciences, Poland \\ 3 Department of Social Sciences and the Humanities, Poznan University of Medical Sci- \\ ences, Poland
}

\begin{abstract}
The aim of this study was to analyse the relationship between the following three assessments: the Neonatal Behavioral Assessment Scale (NBAS), the Alberta Infant Motor Scale (AIMS), and the General Movement Assessment (GMA). 29 patients from the neonatal unit of the Gynecology and Obstetrics Clinical Hospital were examined. The study was conducted between feedings by a person properly trained in the use of the NBAS, the AIMS, and the GMA. The average postmenstrual age of the examined newborns was 35.6 weeks. The average week of gestation was the $29^{\text {th }}$, the average birth weight was $1469 \mathrm{~g}$, and the Median Apgar score in the fifth minute of life was 7. A relationship was found between the AIMS and the NBAS. It was concluded that the Neonatal Behavioral Assessment Scale and the Alberta Infant Motor Scale may be used to determine the motor development of preterm infants. Moreover, it is advisable to use more than one assessment method in order to adjust the intervention.
\end{abstract}

\section{Introduction}

The assessment of the movement of infants should be widespread, especially in the course of early intervention programs. The main goal of the assessment is to monitor the development of a child and to plan his/her therapy goals accordingly. Using scales very often enables the examiner to detect any deficits resulting from a central nervous system disorder. A precise developmental diagnosis should contain an accurate observation of the quality and quantity of movement.

In order to be replicable, a standardized assessment should include a clearly described and defined procedure. According to Hall \& Braid (1986), 
an interpretation of results should also be described in order to make the diagnosis objective. The selection of a standardized test is as important as its implementation. The prognostic value of developmental tests, especially in children in the first few months of life, is limited due to the individualized nature of human development (Auber, 2008).

The Neonatal Behavioral Assessment Scale (NBAS) was developed by Dr. T. Berry Brazelton, Clinical Professor of Pediatrics Emeritus at Harvard Medical School in cooperation with several other specialists. The first edition of the NBAS was published in 1973 by Spastics International Medical Publications in London. According to its authors, NBAS is especially suitable for children born at the typical gestation time and can be used up to the second month of life. Supplementing the assessment with additional items allows it to be used in healthy children who have reached 37 weeks of foetal life and in late premature babies. However, there are studies that describe the application of the NBAS in neonates as early as in the $28^{\text {th }}$ week of gestation (Jesus et al., 2018; Ribas et al., 2019). The authors of the NBAS emphasize that this assessment of behaviour should not be utilized in newborns requiring intensive care. The assessment may be difficult to implement in a child who is not mature enough (Brazelton \& Nugent, 2011). Subsequent editions of the NBAS were supplemented with additional items. The new edition provides evaluation criteria not only for children who are developing normally, but also for high-risk newborns. The new edition also provides guidelines on the clinical use of the scale and emphasizes the possibility of using the tool for the purposes of scientific research.

The Brazelton scale is a neurobehavioral scale whose additional benefit is helping to establish a relationship between parents and children. This tool is composed of 28 behavioural items, each of which employs a nine-point scale. The items regard habituation, social integration, the motor system, state organization and state regulation, the autonomic nervous system, and reflexes. Observations of neurological reflexes (18 items) are assessed on a 4point scale. A further seven items from the NBAS, called "supplementary items", are used to describe the scope of high-risk neonatal behaviour such as the cost of attention, state regulation, and endurance. The examiner may use stimuli such as a flashlight, bell, rattle, or a red ball. They also observe the patient's face. Kwong et al. (2018) indicated that asymmetric tonic neck reflex (ATNR), also an item from the NBAS, may be suggestive of unilateral cerebral palsy (UCP).

Another method of assessing an infant is through the General Movement Assessment (GMA) performed by specialists who utilize Prechtl's method of assessment and diagnosis. The evaluation of the quality of 
General Movements (GMs) at three to four months post-term has been found to have the highest predictive value (Hadders-Algra, 2004). According to Kwong et al. (2018), the specificity of GMs in later cerebral palsy prediction is $91 \%$ (95\% CI 83-95). The method's sensitivity reaches 82 to $100 \%$ at three months post-term (Einspieler et al., 1997). An individual who observes a baby's spontaneous movements may see writhing movements which gradually disappear or "fidgety" GMs when a child is between the ages of six to nine weeks old. Both writhing and fidgety GMs mean that the child's development is normal. If the central nervous system is somehow impaired, then GMs lose their fluency, variability, and complexity. Then, fidgety GMs may be abnormal or absent. A child may present cramped and synchronized movements which may be characterized by a rigid contraction of the limbs. Another alarming movement is chaotic movement. An infant may also present an overall poor skill repertoire, especially if he/she is born preterm, according to Brazelton \& Nugent (2011). The Alberta Infant Motor Scale (AIMS) is a scale used to observe the motor development of a child from the moment of birth up to 18 months of age. This scale was created by Martha Piper and Johanna Darrah in the early 1990s, and its primary purpose is to detect abnormalities that may inhibit a child's motor development. It consists of 58 items of spontaneous antigravity movement performed by an infant in a given position divided on a test sheet in such a way as to evaluate high motility in four positions: pronation (21 items), supination (9 items), in strength (12 items), and in a standing position (16 items). The AIMS assessment evaluates the performed function using a 0-1 point system (yes, no). The number of points attained in all four positions is converted into percentiles for comparison with the age-equivalent in the normative test of typically developing infants. This scale allows the examiner to divide children into three groups: 1) Those developing incorrectly, 2) Those at risk, and 3) Those developing correctly (Liao \& Campbell, 2004).

The AIMS is widely used to assess the motor skills of newborns (Syrengelas et al., 2010) as well as premature babies (Jeng et al., 2000), or children with cerebral palsy (Barbosa et al., 2003). All these scales are valuable. Moreover, the GMA scale has a sensitivity of $60 \%$ and a specificity of $100 \%$ in predicting the scores derived from the AIMS assessment, according to Ricci et al. (2018).

The aim of this study was to analyse the relationship between three assessment methods (NBAS, GMs and AIMS) used for preterm infants in a neonatal unit. 


\section{Materials and Methods}

The assessment of newborns was carried out between feedings by a researcher properly trained in the use of the Neonatal Behavioral Assessment Scale (NBAS), the AIMS, and the Prechtl's Method for the GMA.

Infants with one or more of the following health conditions were excluded: infections, septicaemia, pneumonia, meningitis, osteoarthritis, tumours, fractures or skull bone damage, encephalopathy, hypotension, low heart rate, a state of parasitism, and fatal birth defects (e.g., Edwards syndrome, Patau syndrome, etc.). Infants who were born before eight weeks' postmenstrual age were included. An infant was assessed only if his/her vital signs and overall health condition were considered stable.

Twenty-nine patients were assessed in the neonatal unit of the Gynecology and Obstetrics Clinical Hospital. This study was approved by the Institutional Review Board (consent ref. no. 734/19), and written consent was obtained from the parents or legal guardians of the study's participants prior to the procedure. The study was conducted from October 2017 to March 2018. 18 girls and 11 boys were included in the study group.

The average postmenstrual age of the examined newborns was 35.6 weeks ( $\mathrm{SD} \pm 2.7)$; the average week of gestation was 29.4 weeks $(\mathrm{SD} \pm 3.9)$; the average birth weight was $1469 \mathrm{~g}( \pm 612 \mathrm{~g})$, and the median Apgar score in the fifth minute of life was 7 .

The patients were examined in a single session one time in the neonatal unit. The following items from the NBAS for stable infants were observed and assessed: habituation, social interaction, motor system, state regulation and organization, autonomic nervous system (ANS), reflexes, and supplementary items. For instance, the "habituation" and "social interactive" behaviour of infants, including the ability of the infant to react to environmental stimuli, such as visual or auditory stimuli, both animate (e.g. the voice of mother or father etc.) and inanimate (e.g. a bell, red ball, rattle, etc.) were assessed. Motor system parameters such as "general tone", "activity level", and "pull - to - sit" were assessed. The level of activity of the infant and the regulation of his/her arousal states (e.g. peak of excitement, irritability, lability of states, etc.) were assessed. Most of the NBAS items were assigned 1 to 9 points according to the aforementioned scoring criteria, with a higher score typically indicating better performance, and a lower score indicating inferior performance (there are some items where the opposite is true or where the mid-point is the most optimal score). Reflexes - 18 items - were assessed on a 4-point scale, where a score of 0 indicates 
no reaction, a score of 1 indicates a weak reflex, a score of 2 indicates a modulated response, and a score of 3 indicates an exaggerated reaction. The NBAS was implemented by a researcher formally trained in the administration of the NBAS in Addenbrooke's Hospital in Cambridge, England, who worked professionally in the neonatal unit. The infant being assessed becomes overreactive to stimuli or experiences other medical issues, which may interfere with the implementation of the NBAS assessment. All scoring was performed in accordance with the criteria set out in the NBAS manual (Brazelton \& Nugent, 2011). Another method of assessment was the GMA, which is also a non-invasive examination. This assessment was conducted by observing GMs such as writhing, cramped synchronised movements, or poor repertoire in infants, with all of the infants younger than 8 weeks postmenstrual age. The infants were assessed while lying in a cot in a supine position. The follow-up time for the GMA was approximately 3 to 4 minutes.

The AIMS was based on the observation of movement and scored 1 point for each observed item and 0 points for a lack of movement. The sum of points was converted into percentiles by default and compared with ageequivalent scores.

\section{Results}

The obtained results were subjected to statistical analysis with the use of Statistica 13 (TIBCO Software, Tulsa, USA) and Microsoft Excel 2007. The distributions of the analysed parameters were assessed with the Shapiro-Wilk Test. Both parametric and non-parametric tests were used for the analysis. The level of statistical significance was considered to be $p<0.05$. Considering the complexity of the NBAS scale, in which the sum of all the obtained points lacks relevance (a higher result obtained for certain items does not necessarily mean better performance), only selected packages (i.e. motor system, habituation and social interaction scoring) were used in the data analysis.

In order to verify the research problem, an analysis of the r-Pearson correlation coefficient was performed (Figure 1). The results of the analysis turned out to be statistically significant: $r=0.56 ; p<0.05(p=0.0018)$. This indicates that there exists a relationship between the results obtained using the NBAS scale (taking into account only the sum of points obtained in the "motor system" domain) and the score obtained using the AIMS scale. 
Aleksandra Tabaczyńska et al.

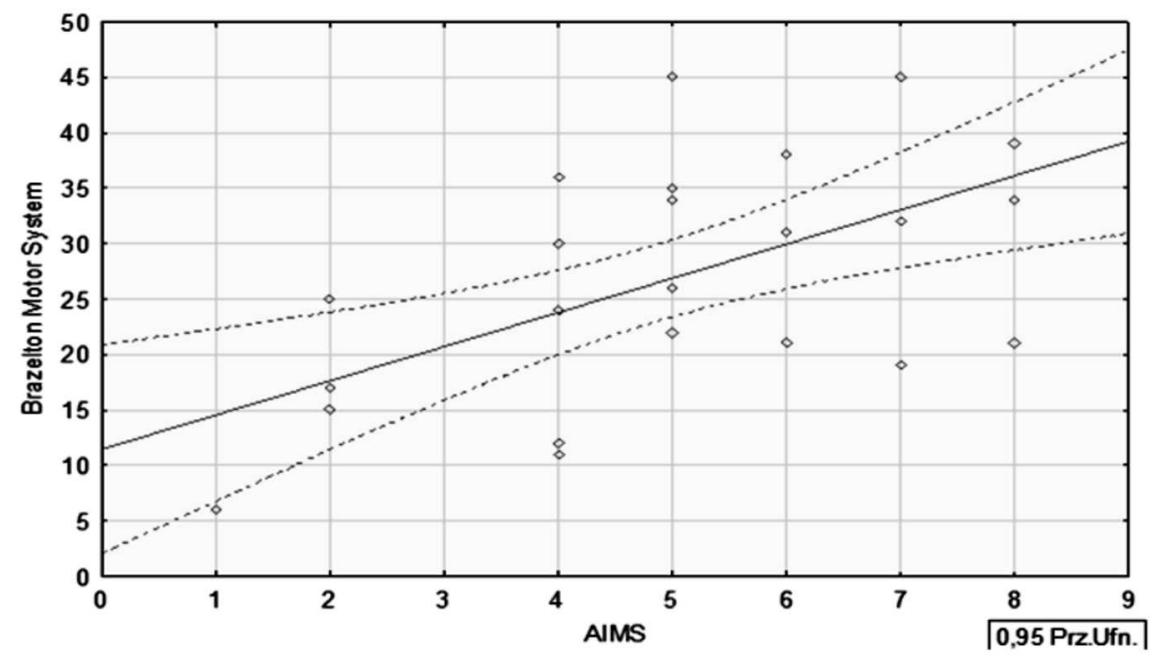

Figure 1. The Alberta Infant Motor Scale vs. the Neonatal Behavioral Assessment Scale (Motor System)

After analysing the rho-Spearman correlation coefficient, a weak correlation $(r=0.42)$, but still a statistically significant finding $(p=0.03)$, was observed between the results obtained in the AIMS and in the "habituation" part of the NBAS scale (Figure 2). The significance of the correlation coefficient was $p=0.03$.

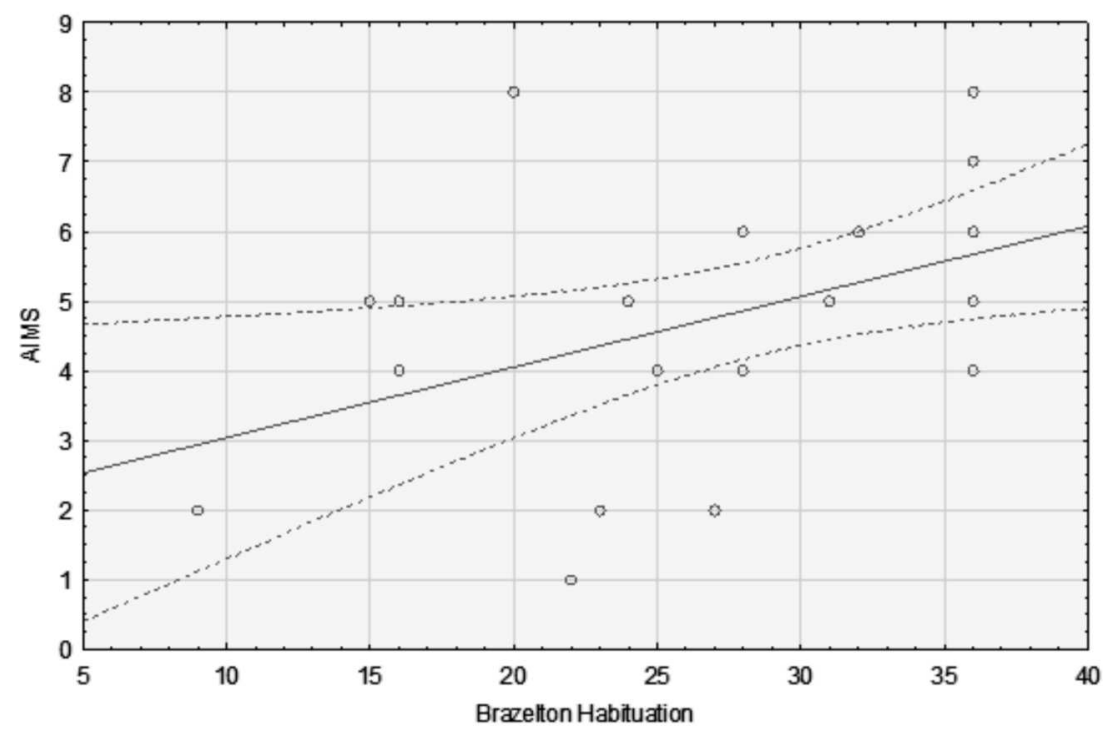

Figure 2. The Alberta Infant Motor Scale versus the Neonatal Behavioural Assessment Scale (Habituation) 
An analysis of Spearman's rank correlation coefficient showed a relationship between the NBAS score for "motor system" and "social interactive" $(r=0.38, p=0.04)$, (Figure 3). Patients presenting a "poor repertoire" in GMs scored fewer points on the NBAS scale in "Motor System" items (Figure 4).

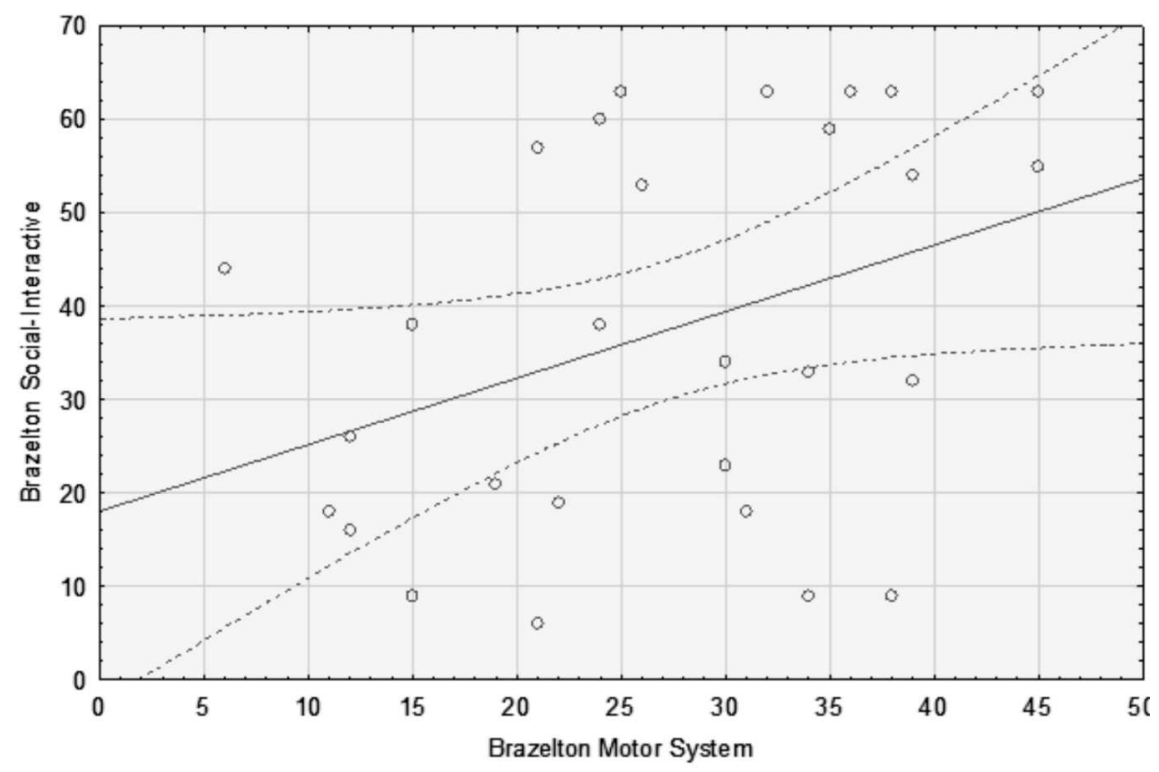

Figure 3. The Neonatal Behavioral Assessment Scale: "Motor System"vs. "Social Interactive"

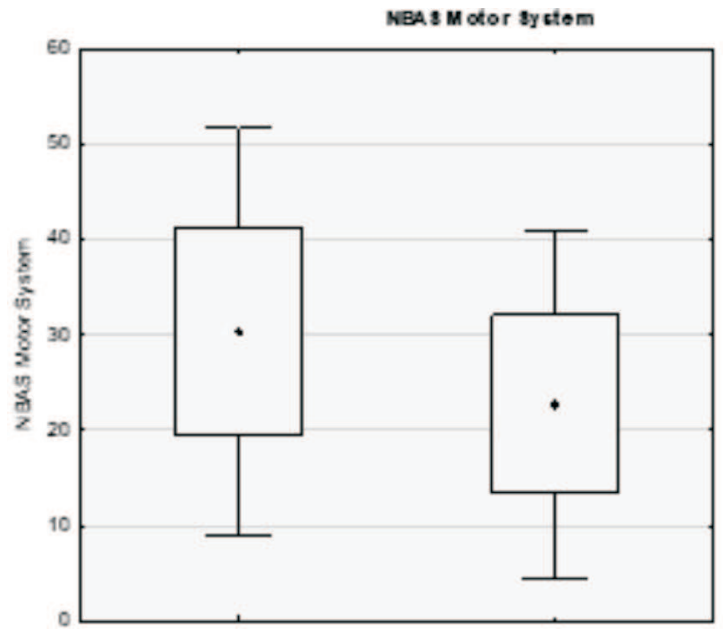

Figure 4. Neonatal Behavioral Assessment Scale (Motor System) vs. General Movements 


\section{Discussion}

Diagnosing and anticipating health problems early in life is crucial to an infant's development. Scales for the assessment of various aspects of development are helpful in finding high-risk areas of concern in children. Some of these scales were created for motor assessment while others focus more on neurobehavioral assessment. These assessments can also be divided into prognostic, comparative, and those that evaluate the developmental potential (Belot et al., 2021).

The Alberta Infant Motor Scale (AIMS) is a scale whose role is to identify delays in motor development or to find its irregularities. It evaluates motor development in relation to age. According to Syrengelas et al. (2010), it is a quantitative tool, evaluating only the presence or lack of motor ability, without the possibility to qualitatively analyse changes occurring during therapy of children with impaired development.

It may be used by physiotherapists who work with children and focus on the child's motor skills. In order to carry out this assessment, therapists most often use some kind of physical handling. The infant's position may change from supination to pronation, a sitting position (traction), or even standing. Additionally, the observation of the mechanism of antigravity and muscle tone may prove too burdensome for newborns in intensive care units. Moreover, such examination may not be possible in children requiring oxygen therapy or multi-channel monitoring of their states (Brazelton \& Nugent, 2011).

This makes the assessment of high-risk neonates more demanding. It is important to minimize the time spent on the examination and to limit the tasks that are required for the assessment - especially in the case of preterm infants.

The assessment scale that focuses on neurobehavior is the NBAS created by T. Berry Brazelton. According to Brown \& Spittle (2014), it is the first tool designed specifically to assess a child's competences in a social context. The observation of habituation, individual differences in the behaviour of infants, as well as the awareness of the skills and abilities of the mentee influence the relationship between the carer, the child, and the parents of a neonate.

As reported by Brazelton \& Nugent (2011), the NBAS focuses on the social interaction skills of the infant, his/her ability to function in the environment, as well as the ability of the infant to self-soothe and adjust his/her behaviour when presented with different stimuli. It is apparent that the child is ready for social interaction when his/her motor and autonomic systems 
are working properly. This assumption is reflected in the part of the scale devoted to the study of motor skills and reflexes.

The General Movements Assessment (GMA) is an accurate and reliable method of evaluation of an immature nervous system. Researchers such as Bouwstra et al. (2010), Darsaklis et al. (2010), and Büşra Kepenek-Varoll et al. (2016) show that the analysis of GMs plays a huge role in the prognosis of neurodevelopmental disorders and the identification of newborns who may later develop atypically.

The predictive value of the assessment of GMs depends on the age when the assessment is carried out for an infant as well as on the obtained result. Although the prognosis is most effective at the age of 3 to 4 months post-delivery, even infants 36-38 weeks' old postmenstrual age (PMA) who show abnormal GMs (cramped synchronized or chaotic, poor repertoire) are at the risk of developing cerebral palsy (70\%-85\%), according to HaddersAlgra (2004).

The NBAS scale has been used in numerous studies. Alvarez-Garcia et al. (2015) compared a group of preterm babies with a group of children born on time and found that premature babies received a lower neurobehavioral score and a surprisingly higher score in the habituation domain. Amini et al. (2016) conducted a study to determine if there exists a relationship between motor system function and behavioural performance. They examined children with low birth weight between thirty-six weeks' PMA to 2 months of age. They observed a relationship between the score received in the social relations and the motor domain. Their observations support the results of this study concerning social interactions with points obtained in the motor section of the NBAS. A statistically significant correlation was found between those two domains of the NBAS.

An assessment of an infant's neurobehavior may be beneficial, especially for high-risk newborns. This finding is supported by the correlation between scores from the NBAS (habituation items) and the scores obtained in the study using the AIMS scale. The statistically significant correlation $(r=0.42, p=0.03)$ between an infant's ability to react to sensory stimulation and the level of the infant's motor development may also lead to his/her ability to self-regulate and contributes to self-development and maturation.

An analysis of the relationship between the motor section of the NBAS scale and the AIMS study reveals the existence of a statistically significant $(p=0.002)$ mean correlation $(r=0.56)$. This can be explained by the fact that both scores relate directly to the motor skills that the child presents. The relationship between the obtained results show the possibility to choose the appropriate scale, taking into consideration the condition of the examined child. 
According to the GMA, the average number of children presenting a "poor repertoire" had lower score in motor cluster and social cluster in the NBAS. From the analysis of the average number of points obtained in the motor domain with the division into groups of children presenting "writhing movements" and a "poor repertoire", it appears that the score in this range has the highest predictive value (Hadders-Algra, 2004). Despite the observed trend, the use of the Brazelton scale to predict the future development of newborns is not justified, in contrast to the GMA (Kwong et al., 2018).

The Brazelton scale is an excellent tool for describing the threshold of tolerance to accepted and processed stimuli, determining the costs of reactions for a child, assessing the autonomic nervous system, reflex behaviour, orientation reactions, and motor skills. The system is suitable both for children born full term and preterm infants in intensive care units. Numerous studies have used the NBAS scale to evaluate children starting from the $36^{\text {th }}$ week of gestational age (Amini et al., 2016; Malak et al., 2021; Ohgi et al., 2003). As it turns out, the scale was also beneficial for children born much earlier - the youngest of the examined participants was 32 weeks' PMA. This is why it has been decided that the scale should be used for the children in our research group.

Despite the fact that the NBAS focuses on behavioural assessment, some of its aspects can be used to analyse motor development. However, it should be kept in mind that the presented relationships are not strong, and therefore the best solution seems to be the assessment of children using more instruments, such as the GMA or AIMS. None of the newborn assessment tools presented in the paper is capable of assessing all aspects of development.

\section{Conclusions}

It is possible to use different types of assessment scales, such as the NBAS, the GMA, and the AIMS, depending on the type of information needed in the neonatal unit. Owing to the scales, and thanks to the clinicians and caregivers of preterm infants, the authors of this study had the opportunity to become acquainted with each participant's behaviour, including motor system, social interaction skills, and habituation. It is possible to assess the features of the motor system in preterm infants via the AIMS and NBAS. These assessment scales can be used to help plan and apply an appropriate therapy as early as possible. 
Alvarez-Garcia, A., Fornieles-Deu, A., Costas-Moragas, C., \& Botet-Mussons, F. (2015). Neurobehavioral conditions and effects of gender, weight and severity in preterm infants according to the Neonatal Behavioral Assessment Scale. Anales de Psicologia, 31(3), 818-824. doi: http://dx.doi.org/10.6018/analesps. 31.3.170181

Amini, M., Aliabadi, F., Alizade, M., Kalani, M., \& Qorbani, M. (2016). The Relationship between Motor Function and Behavioral Function in Infants with Low Birth Weight. Iran J Child Neurol., 10(4): 49-55. doi: https://doi.org/10. $22037 /$ ijcn.v10i4.10965

Auber, E. (2008). The variability of human growth and development. In J. S. Tecklin (Ed.), Pediatric Physical Therapy. Fourth edition (pp. 17-20). Lippincott Williams \& Wilkins, Philadelphia. https://doi.org/10.1080/0194263080230 9651

Barbosa, V. M., Campbell, S. K., Sheftel, D., Singh, J., \& Beligere, N. (2003). Longitudinal performance of infants with cerebral palsy on the Test of Infant Motor Performance and on the Alberta Infant Motor Scale. Physical \& Occupational Therapy in Pediatrics, 23(3): 7-29. doi: 10.1080/j006v23n03_02

Belot, R. A., Bouteloup, M., Mariage, A., Candilis-huisman, D., Mottet, N., \& Mellier, D. (2021). Relevance of Neonatal Behavior Assessment Scale for Infants With Somatic Disorders: Comparison on One Matched Group of Control. Frontiers in Pediatrics, 8, 506384. doi: https://doi.org/10.3389/fped.2020. 506384

Bouwstra, H., Dijk-Stigter, G. R., Grooten, H. M., Janssen-Plas, F. E., Koopmans, A. J., Mulder, C. D., van Belle, A., \& Hadders-Algra M. (2010). Predictive value of definitely abnormal general movements in the general population. Developmental Medicine \& Child Neurology, 52(5), 456-461. doi: 10.1111/j.1469-8749.2009.03529.x

Brazelton, T. B., \& Nugent, J. K. (2011). Neonatal Behavioral Assessment Scale 4th Edition. Mac Keith Press, London.

Brown, N., \& Spittle, A. (2014). Neurobehavioral Evaluation in the Preterm and Term Infant. Curr Pediatr Rev., 10(1), 65-72. doi: 10.2174/157339631001140 408121310

Büşra, K-V., Mine, Ç., Zeynep, İ., Burak, T., Emine, E., \& Asuman, Ç. (2016). The comparison of general movements assessment and neurological examination during early infancy. The Turkish Journal of Pediatrics, 58, 54-62. doi: 10.24953/turkjped.2016.01.008

Darsaklis, V., Snider, L. M., Majnemer, A., \& Mazer, B. (2010). Predictive validity of Prechtl's Method on the Qualitative Assessment of General Movements: a systematic review of the evidence. Developmental Medicine \& Child Neurology, 53(10), 896-906. doi: 10.1111/j.1469-8749.2011.04017.x 
Einspieler, C., Prechtl, H. F., Ferrari, F., Cioni, G., \& Bos, A. F. (1997). The qualitative assessment of general movements in preterm, term and young infants-review of the methodology. Early Hum Dev., 50(1), 47-60. doi: 10.1016/s0378-3782(97)00092-3

Hadders-Algra, M. (2004). General movements: a window for early identification of children at high risk for developmental disorders. The Journal of Pediatrics, 145(2), 12-18. doi: 10.1016/j.jpeds.2004.05.017

Hall, D. M., \& Baird, G. (1986). Developmental tests and scales. Archives of Disease in Childhood, 61, 213-215. doi: 10.1136/adc.61.3.213

Jeng, S. F., Yau, K. I., Chen, L. C., \& Hsiao, S. F. (2000). Alberta infant motor scale: reliability and validity when used on preterm infants in Taiwan. Physical Therapy, 80(2), 168-178. doi:10.1155/2018/2197163

de Jesus, V. R., de Oliveira, P. M. N., \& Azevedo, V. M. G. O. (2018). Effects of hammock positioning in behavioral status, vital signs, and pain in preterms: A case series study. Brazilian J. Phys. Ther., 22, 304-309. doi: https://doi. org/10.1016/j.bjpt.2018.03.002

Kwong, A. K., Olsen, J. E., FitzGerald, T. L., Doyle, L. W., Cheong, J. L. \& Spittle, A. J. (2018). Spontaneous infant movements that predict later cerebral palsy: reply to Hadders-Algra and Philippi. Dev Med Child Neurol., 60, 1289 1289. doi: $10.1111 /$ dmcn. 14036

Liao, P. M., \& Campbell, S. K. (2004). Examination of the Item Structure of the Alberta Infant Motor Scale. Pediatric Physical Therapy., 16(1): 31-38. doi: 10.1097/01.PEP.0000114843.92102.98

Malak, R., Sikorska, D., Rosołek, M., Baum, E., Mojs, E., Daroszewski, P., Matecka, M., Fechner, B. et al. (2021). Impact of umbilical cord arterial pH, gestational age, and birth weight on neurodevelopmental outcomes for preterm neonates. PeerJ, 9:e12043. doi: https://doi.org/10.7717/peerj.12043

Ohgi, S., Arisawa, K., Takahashi, T., Kusumoto, T., Goto, Y., Akiyama T., \& Saito, H. (2003). Neonatal behavioral assessment scale as a predictor of later developmental disabilities of low birth-weight and/or premature infants. Brain 6 Development, 25(5), 313-321. doi: 10.1016/s0387-7604(02)00233-4

Ribas, C. G., Andreazza, M. G., Neves, V. C. \& Valderramas, S. (2019). Effectiveness of hammock positioning in reducing pain and improving sleepwakefulness state in preterm infants. Respir Care., 64, 384-389. doi: https:// doi.org/10.4187/respcare.06265

Ricci, E., Einspieler, C., \& Craig, A. K. (2018) Feasibility of Using the General Movements Assessment of Infants in the United States. Physical \& Occupational Therapy In Pediatrics, 38(3), 269-279. doi: 10.1080/01942638.2017.13 95380

Syrengelas, D., Siahanidou, T., Kourlaba, G., Kleisiouni, P., Bakoula, C., \& Chrousos, G. P. (2010). Standardization of the Alberta infant motor scale in fullterm Greek infants: Preliminary results. Early Human Development, 86(4), 245-249. doi: 10.1016/j.earlhumdev.2010.03.009 